\title{
Formalized reliability analysis during the electric power systems modes management
}

\author{
Sigitov O.Yu. ${ }^{1,{ }^{*}}$, Chemborisova N.Sh. ${ }^{1}$, and Chernenkov I.D. ${ }^{1}$ \\ ${ }^{1}$ National Research University Moscow Power Engineering Institute, 111250, Moscow, Russia
}

\begin{abstract}
The current problem of the selection of the electric power system control nodes, which can be used to solve multicriterial problems of the consumers reliable supply with the electric power of the necessary quality of maximum efficiency is investigated. At the same time, a new method of making decisions while choosing electric power system control nodes is proposed. It is necessary to take into account a set of different types of indicators which characterize different properties of these nodes.
\end{abstract}

\section{Introduction}

Modern problems of the electric power industry are solved taking into account a large number of various data (often heterogeneous), influencing factors and decisionmaking criteria that do not always agree with each other. This requires new approaches for solving the known problems of estimating the electric power systems (EPS) modes parameters, calculating and analyzing such modes, their reliability, stability, etc. In this regard, the modes management with the provision of the indicators having normative or boundary values, the excess of which is not allowed in the normal operation of the electric power systems, turns into a multicriterial task.

One of the components of the regime management overall task is to ensure reliability. For example, under market conditions the consumers power supply reliability, which directly depends on the structural and regime components, becomes a commodity. The fee is charged for the power supply reliability while connecting consumers electrical installations to the electric networks and certain compensation may be provided for the violation in the excess of the established standard. This compensation can be specified in the power supply contracts. In this regard, the modes management ensuring reliable power supply becomes a technical and economic task, the initial data and solution of which must be obtained and taken into account during the electric power system current operation, that is quickly enough.

\section{Multicriterial reliability problem solution}

Taking into account different goals and criteria, it becomes necessary to make a decision on the choice of control nodes for which the control influence will be the most effective. In this case the assessment should be carried out taking into consideration different types of data, which have different units of measurement, scales and measurement scales. The general theory of such solutions is described in Source [1], where it is noted that "analytic hierarchy process seems to be more justified by solving multicriterial problems in a complex environment with hierarchical structures including both tangible and intangible factors than the approach based on linear logic, which may not lead to the best solution, since in this case the possibility of making compromises among factors lying in different chains of logical thinking may be lost". The use of the analytic hierarchy process allows to include all the knowledge and imagination available on the problem under consideration in the hierarchy $[1,2]$. In addition, it is important to have simple and effective methods for preliminary analysis of the problem. These methods are based on dependencies among some generalized indicators and scheme parameters, as well as parameters of the electric power system mode. They allow to rank the electric network nodes and determine priorities for further analysis and decision-making.

As an example, let's consider the problem of choosing the optimal power and places of reactive power compensation devices installation in the network organization nodes. The research shows that reactive power compensation must be performed at all voltage levels, both for consumers and in the electric networks. At the same time, there are other important factors in real tasks that influence the choice of the reactive power compensation devices installation location. The simplest, but rather effective method for solving this problem is to assess the ability of nodes to maintain voltage relatively well when their load changes per conventional unit "hardness". The electric power system nodes with the opposite property are called "sensitive" nodes. This problem is solved and illustrated in a number of papers using the Jacobi matrix and the "balance-conductivity" method [3-6]. The essence of the method is a fairly simple and easily implemented procedure for assessing the difference between the imaginary components of node intrinsic conductivity and sum of nodes mutual

\footnotetext{
*Corresponding author: olegsigitov@gmail.com
} 
conductivities of the electric power system studied scheme [4,5]. With the same signs for all balance conductivities, that have a capacity character, the minimum values of this indicators module show a high degree of the node "sensitivity", the largest values point out its "hardness". For different signs of the balance conductivities, it should be taken into account that the largest modulo inductive components characterize the most sensitive nodes and the largest capacitive ones characterize hard nodes. The use of sensitive nodes as control nodes gives good results in practice, since their voltage is more sensitive to changes of the load reactive component. At the same time, the value of the control effect may not be large with a positive effect of regulation, both in simple and complex heterogeneous networks [35].

Another indicator is the amount of three-phase short circuit (SC) current is also an important characteristic because after installing reactive power compensation devices, the degree of the nodes hardness increases and $\mathrm{SC}$ currents rise. The value of the three-phase short circuit current in sensitive nodes is noticeably less than in hard nodes, which is shown in sufficient detail acording the results of the Moscow power system studies [4]. In this case, there is a fairly strong stochastic relationship between the three-phase SC currents in 11 nodes of the electrical network, which are taken, for example Source [6], and the values of their balance conductivity with a correlation coefficient $\mathrm{r}=0.902$. Thus, the formal statement that the short circuit currents in the hard nodes of the electric power system are significantly greater than in the sensitive ones has been practically confirmed in the stochastic model with a high correlation coefficient.

In addition to these indicators, there are other indicators that determine the consumers power supply reliability and the efficiency of active and reactive power distribution in network nodes, etc.

One of these factors is the loss of active power and energy in the network. It is shown in Article [7] that during optimization of the electrical modes, taking into account active power losses in the network and using the balance-conductivity method, it is possible to obtain a voltage increase by an average of $5.4 \%$ and a decrease in the network total losses by $14 \%$ in relation to the basic calculation option while controlling in the two most sensitive nodes. A change in the other nodes reactive power of the network by the same amount leads to less efficient mode control [6,7], an increase in the volume of control action that is control in sensitive nodes will be close to optimal one. Active losses change in the network also changes the capacity balances in this network, which must be taken into account during the balance reliability analysis [8]. In this case a preliminary analysis of balances allows to take into consideration the influence of actions in the most sensitive nodes on the formation of balances in the network, as well as to evaluate the effectiveness of modes management in various network nodes.

The node voltage sensitivity coefficient to changes in active and reactive loads determines the degree of its hardness (sensitivity), which significantly affects its load capacity, controllability, the short circuit currents value, etc.
The number of overhead transmission lines connected to the node (connections among nodes), which is taken into account while calculating structural reliability, is also an indirect indicator of the node hardness degree, the consumers power supply reliability, etc.

The average voltage value in all nodes of the system under study after the installation of the reactive power compensation devices in a particular node characterizes the effectiveness of this event in combination with the voltage variation indicators of the standard deviation and the average relative increase. The higher the average voltage value and the lower the standard deviation the higher the efficiency of the reactive power compensation devices installation in the node under consideration. It should be added that the voltage value in the node significantly affects all the important indicators of the electric power system operating mode: it increases the throughput capacity and stability (static and dynamic), changes (in general) the active and reactive power losses, changes the short circuit currents, and affects the electrical energy quality. Then all these indicators must be taken into account when forming the algorithm and data for the analytic hierarchy process.

For example, let's consider these provisions in the method of effective arrangement of the reactive power compensation devices, taking into account various influencing factors while analyzing a complex network with a large number of closed circuits and energy sources. A scheme of the electric power system model fragment used in the «Finist» dispatcher mode simulator is shown in Fig. 1 [9].

The fragment under study includes a complex branched electrical network with the voltage of $110 \mathrm{kV}$ and $220 \mathrm{kV}$. The network receives electrical energy from two thermal power plants of different capacities, and there are also connections to the external electric power system via $220 \mathrm{kV}$ transmission lines and half of the required electrical energy for the network under study is received via them. In this mode, the active power of consumers is equal to $1070 \mathrm{MW}$, the reactive power is $406 \mathrm{MVAr}$ and is unevenly distributed among the system nodes.

We will sequentially consider the installation of the reactive power compensation devices of the same capacity in each of the studied nodes, except substations of the electric power plants and connection points to the system, node 7 of the external EPS, where two synchronous compensators are already installed. It is also taken into account that the $110 \mathrm{kV}$ bus sections normally operate with the switched-on sectional power switches, what increases the short circuit currents (up to acceptable values), but equalizes the voltage levels.

Partially the integral indicators calculations results obtained using the analytic hierarchy process and taking into account all the above-mentioned factors are presented in Table 1. The best result (the most effective management using the reactive power compensation devices to improve the conditions for maintaining the consumers power supply reliability) is the result with the highest integral indicator. 


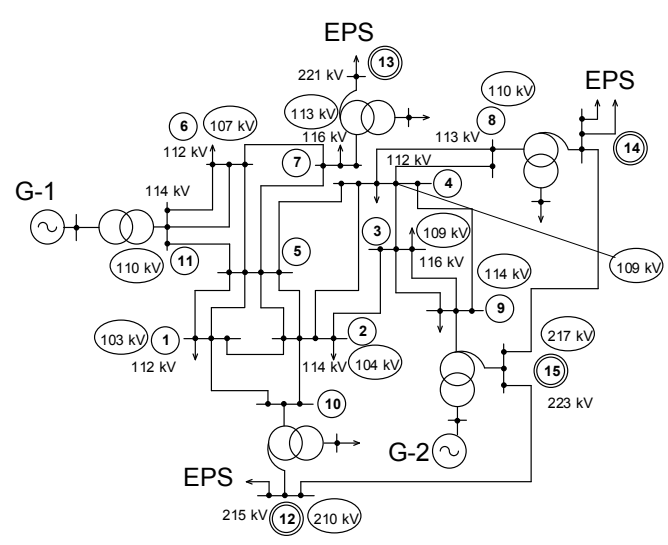

Fig. 1. Scheme of the $220 \mathrm{kV}$ and $110 \mathrm{kV}$ network fragment under study.

To evaluate the obtained results integral indicators of the reactive power compensation devices installation priority in the nodes of the network under study, total losses of active power in the network, the nodes balance conductivity indicators and three-phase SC currents are compared in Table 1. There is a close stochastic relationship between these indicators and the node ranks integral indicators. For example, the decrease in the integral index of node ranks is directly proportional to the decrease in their hardness index and inversely proportional to the short-circuit current value.

Table 1. Indicators of the studied nodes.

\begin{tabular}{|c|c|c|c|c|}
\hline $\begin{array}{c}\text { Node } \\
\text { no. }\end{array}$ & $\begin{array}{c}\text { Integral } \\
\text { indicator }\end{array}$ & $\begin{array}{c}\Delta \mathbf{B}, \\
\text { microSm }\end{array}$ & $\begin{array}{c}\mathbf{S C} \\
\text { current, } \\
\mathbf{k A}\end{array}$ & $\begin{array}{c}\Delta \mathbf{P}, \\
\mathbf{M W}\end{array}$ \\
\hline 1 & 0,63 & 574 & 11,61 & 33,8 \\
\hline 2 & 0,83 & 997 & 16,76 & 28,16 \\
\hline 3 & 0,47 & 460 & 15,6 & 29,43 \\
\hline 4 & 0,53 & 847 & 16,72 & 29,62 \\
\hline 5 & 0,87 & 1142 & 17,33 & 29,01 \\
\hline 6 & 0,5 & 390 & 9,48 & 34,71 \\
\hline 7 & 0,63 & 930 & 15,90 & 31,26 \\
\hline 8 & 0,53 & 572 & 14,52 & 29,74 \\
\hline 10 & 0,6 & 612 & 12,08 & 34,01 \\
\hline
\end{tabular}

The calculations have shown that the lowest values of active power losses in the network correspond to the maximum values of the integral indicator and the value $\Delta B_{i}$

$i$. Then we can conclude that the network mode most effective control is possible when the reactive power changes in the node with the highest integral index. Keep in mind, that the reactive power compensation devices arrangement affects not only the reduction of power and electricity losses in the network and the voltage level, but also other indicators in the EPS. For example, such an important property of the EPS as its stability depends on the magnitude and phase of the voltage in the node and must be preserved for small and large disturbances in the system. Thus, after a preliminary analysis of the network scheme and the base load of its nodes information by the balance-conductivity method, a preliminary conclusion about the ranked ratio among the nodes integral indicators can be made using the analytic hierarchy process.

While analyzing the various EPS functioning reliability, the necessity of the nodes account, which load has a strongly expressed probabilistic character, may occur. For example, wind power plants (wind farms), due to the technological features of their operation, depending on wind speed and climatic conditions of the area, change the power output and the power balances formation, which affects the active power optimal distribution among electric power plants in the process of continually and randomly electricity changing for the consumer. In this regard, the task of formalized selection of the control nodes most rational list and the control action amount is more complicated. The proposed sequence of analysis of the circuit-mode situation for the test scheme shown in Fig. 2 has the following form.

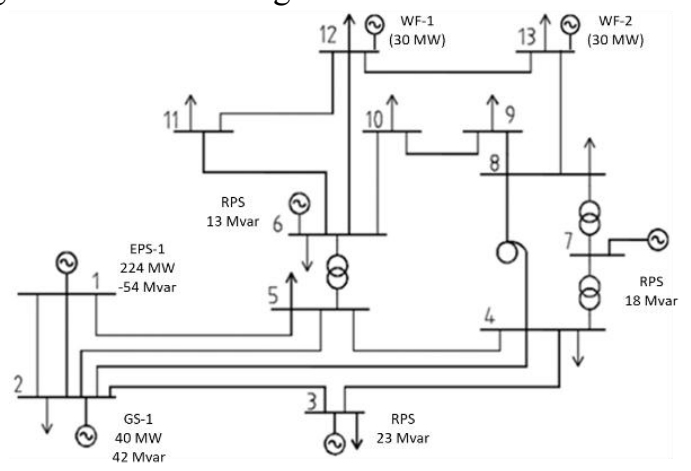

Fig. 2. Test scheme.

For each day, a schedule of the electric power system load, which must be distributed among the electric power plants, is projected per each day. The certain type fuel amount required by a particular power plant for the production of electric energy is calculated in accordance with this schedule [10]. At the same time, one of the management tasks of the local energy complex, consisting of traditional power plants and wind farms, is to optimize the traditional power plants operating generators, since EPS regime economic indicators depend on this [11]. Since the overall objective of optimization is to obtain a minimum cost for the electric energy production and distribution, it is necessary to distribute the load among power plants and units in the most rational and economical way.

The calculation of the steady-state mode, the power plants operating equipment selection optimization and sensitive nodes determination are carried out for the scheme (shown in Fig. 2), which has two generation sources and three reactive power sources. The network scheme parameters are specified in Source [12]. Wind farms are located in nodes 12 and 13 with an installed capacity of $30 \mathrm{MW}$ each. In practice wind farms are used primarily as a source of active power. Despite this, the power factor $(\cos \varphi)$ in most cases is about $0.9[13,14]$, so the wind farm calculations are taken into account in the generating reactive power mode corresponding to $\cos \varphi=0.9$. The wind farm integration into EPS nodes will 
lead to its configuration change and heterogeneity that in its turn causes the appearance of EPS nodes, in which the load change can lead to significant voltage deviations. In this regard, the balance conductivity method is used to analyze the impact of the wind farms installation on the operating parameters in the power plant nodes, wind farms are installed in sensitive nodes.

The calculation is performed in two stages:

1. At the first stage, the calculation is carried out without a wind farm including:

1.1. Optimization of the thermal power plants operating equipment selection;

1.2. Calculation of the steady-state mode (maximum loads);

1.3. Definition of sensitive and hard nodes;

2. At the second stage, calculations are made taking into account the wind farms installation in sensitive nodes under different operating modes similar to paragraphs 1.11.3 .

The calculation results are presented in Table 2 and 3 for the scheme without wind farms and taking into account the wind farms operation with different loads due to the changing of the installed capacity utilization coefficient (ICUC) over time. Therefore, the highest loads mode calculation must be supplemented with a wind farms generation change. The ICUC of modern mainland wind farms is about $30-35 \%$, and offshore wind farms is about $35-55 \%$ [15]. In this example the wind farm load was assumed to be equal to $100 \%, 40 \%, 20 \%, 10 \%$.

Table 2. Change of $\Delta \mathrm{B}$ in the network nodes in the highest loads mode at different wind farms ICUC.

\begin{tabular}{|c|c|c|c|c|c|}
\hline \multicolumn{2}{|c|}{$\begin{array}{l}\text { Mode without } \\
\text { wind farms }\end{array}$} & \multicolumn{2}{|c|}{$\begin{array}{c}\text { Mode with wind } \\
\text { farms (ICUC } \\
100 \%)\end{array}$} & \multicolumn{2}{|c|}{$\begin{array}{c}\text { Mode with wind } \\
\text { farms (ICUC } \\
40 \%) \\
\end{array}$} \\
\hline $\begin{array}{l}\dot{g} \\
\dot{0} \\
\dot{0} \\
\dot{z}\end{array}$ & $\stackrel{\vartheta}{\vartheta}$ & $\begin{array}{l}\dot{g} \\
\dot{0} \\
\dot{0} \\
\dot{Z}\end{array}$ & $\stackrel{*}{\vee}$ & $\begin{array}{l}\dot{\Xi} \\
\dot{\Xi} \\
\dot{0} \\
\dot{z}\end{array}$ & $\stackrel{\vee}{\vee}$ \\
\hline 8 & $-0,2648$ & 8 & $-0,2648$ & 8 & $-0,2648$ \\
\hline 4 & $-0,1363$ & 4 & $-0,1363$ & 4 & $-0,1363$ \\
\hline 5 & $-0,0091$ & 5 & $-0,0091$ & 5 & $-0,0091$ \\
\hline 1 & $-0,0016$ & 12 & $-0,0019$ & 1 & $-0,0015$ \\
\hline 12 & $-0,0006$ & 13 & $-0,0018$ & 12 & $-0,0011$ \\
\hline 9 & $-0,0005$ & 1 & $-0,0012$ & 13 & $-0,0010$ \\
\hline 3 & $-0,0005$ & 9 & $-0,0006$ & 9 & $-0,0006$ \\
\hline 13 & $-0,0005$ & 3 & $-0,0005$ & 3 & $-0,0005$ \\
\hline 2 & $-0,0003$ & 2 & $-0,0003$ & 2 & $-0,0003$ \\
\hline 11 & $-0,0002$ & 11 & $-0,0002$ & 11 & $-0,0002$ \\
\hline 10 & $-0,0002$ & 10 & $-0,0002$ & 10 & $-0,0002$ \\
\hline 6 & 0,0165 & 6 & 0,0166 & 6 & 0,0165 \\
\hline 7 & 6,0496 & 7 & 6,0553 & 7 & 6,0513 \\
\hline
\end{tabular}

Table 3. Change of $\Delta \mathrm{B}$ in the network nodes in the highest loads mode at different wind farms ICUC.

\begin{tabular}{|c|c|c|c|c|c|}
\hline \multicolumn{2}{|c|}{$\begin{array}{l}\text { Mode without } \\
\text { wind farms }\end{array}$} & \multicolumn{2}{|c|}{$\begin{array}{c}\text { Mode with wind } \\
\text { farms (ICUC } \\
20 \%) \\
\end{array}$} & \multicolumn{2}{|c|}{$\begin{array}{c}\text { Mode with wind } \\
\text { farms (ICUC } \\
10 \%) \\
\end{array}$} \\
\hline 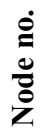 & $\stackrel{\vartheta}{\gamma}$ & $\begin{array}{l}\dot{\Xi} \\
\stackrel{0}{0} \\
\text { Z }\end{array}$ & $\stackrel{\vartheta}{\gamma}$ & $\begin{array}{l}\dot{\Xi} \\
\stackrel{\Xi}{0} \\
\text { Ż }\end{array}$ & $\stackrel{\vartheta}{\psi}$ \\
\hline 8 & $-0,2648$ & 8 & $-0,2648$ & 8 & $-0,2648$ \\
\hline 4 & $-0,1363$ & 4 & $-0,1363$ & 4 & $-0,1363$ \\
\hline 5 & $-0,0091$ & 5 & $-0,0091$ & 5 & $-0,0091$ \\
\hline 1 & $-0,0016$ & 1 & $-0,0016$ & 1 & $-0,0016$ \\
\hline 12 & $-0,0006$ & 12 & $-0,0009$ & 12 & $-0,0007$ \\
\hline 9 & $-0,0005$ & 13 & $-0,0007$ & 13 & $-0,0006$ \\
\hline 3 & $-0,0005$ & 9 & $-0,0006$ & 9 & $-0,0006$ \\
\hline 13 & $-0,0005$ & 3 & $-0,0005$ & 3 & $-0,0005$ \\
\hline 2 & $-0,0003$ & 2 & $-0,0003$ & 2 & $-0,0003$ \\
\hline 11 & $-0,0002$ & 11 & $-0,0002$ & 11 & $-0,0002$ \\
\hline 10 & $-0,0002$ & 10 & $-0,0002$ & 10 & $-0,0002$ \\
\hline 6 & 0,0165 & 6 & 0,0165 & 6 & 0,0165 \\
\hline 7 & 6,0496 & 7 & 6,0503 & 7 & 6,05000 \\
\hline
\end{tabular}

In accordance with the presented results, it becomes clear that the wind farm installation will lead to a change in the difference between its own conductivity and the sum of mutual conductivities, that is, an increase in the node hardness, which will help maintain the voltage in the nodes under consideration and normalize the network voltage. The analysis of the obtained results allows to conclude that the distribution of generation sources in load nodes, in particular the wind farms location, has a positive effect on the electric power system parameters. The calculations results show moving up of the ranked list of the nodes, in which wind turbines are installed, that is, increasing the hardness of the scheme. At the same time, the more wind farm generated power the more noticeable the effect is.

Also, the wind farms integration into electric power systems has a positive impact on power losses in the electric network. It is noted in the sources $[16,17]$, that the wind farms distribution in EPS nodes allows to reduce power losses in the electric network. The losses reduction is achieved due to the generation sources close location to electric energy consumers, which reduces power flows through transmission lines from traditional power plants.

In this way, wind farms within EPS can create competitive solutions providing some advantages. Since wind power is a part of the distributed generation, in the current situation of EPS topology, this is an advantage for ensuring reliability in the sphere of the consumers power supply. The effect is achieved by location of the wind farms not only in places with the most favorable natural, climatic and geographical conditions, but also in EPS sensitive nodes (as an additional condition), thereby increasing their hardness and, as a result, reliability. Accounting for wind farms using in the form described above can also be carried out while calculating integral 
indicators, since the following characteristics are used: nodes voltage, active power losses in the network, $\Delta \mathrm{B}$.

\section{Conclusion}

1. Since the integral indicators obtained by the analytic hierarchy process allow to identify the control nodes that will be the most effective (control organization close to the optimal one) in ensuring the consumers power supply reliability, taking into account the structural and regime components, this approach is quite justified. For the ranked nodes list a formalized preliminary estimation balance-conductivity method can be used. The refined calculations are performed using the analytic hierarchy process.

2. The balance-conductivity method was tested on the electric power system multi-node high-voltage network fragment used in the mode dispatcher simulator «Finist». The simplicity of calculations and the control parameters high accuracy determination were noted, which allow using the method on mode simulators.

3. It is possible to apply analytic hierarchy process and balance-conductivity method successfully in the problem of choosing the optimal location for wind farms installation, since it allows to take into account not only climatic features, but also the electric network scheme parameters. At the same time, the greater the generated power of the installed wind farms, the more noticeable the positive effect (increasing the hardness of the scheme and consumers power supply reliability).

4. The joint application of the analytic hierarchy process and balance-conductivity method in multi-criteria problems of electric networks control modes of various complexity is proposed to improve the consumers power supply reliability.

\section{References}

1. Saati T. Making decisions. Analytic hierarchy process. Moscow: Radio and Communications. 1993.-278 p.

2. Farhatzadeh E.M. etc. Methods and algorithms for comparing and ranking the reliability and efficiency of electric power system objects operation // Electricity, No. 8, 2017. Pp. 10-12.

3. Baranov I.L., Chemborisova N.Sh. Determination of the electric power system nodes sensitivity at the main frequency and higher harmonics // Electricity, No. 8, 2013. Pp. 15-20.

4. Chemborisova N.Sh., Frolov O.V., Baranov I.L., Bashirov I.N. Use of generalized scheme indicators in the analysis of the electric power systems modes // Vestnik MEI, №1, 2015. S. 6673.

5. Chenborisova N.Sh. Accounting for hardness in the evaluation of reliability of the electric power systems operation. Collection of works of the IXth Intern. scientific and technical Conf.
Energy, computer science, innovation - 2019. In 2 t. T 1. - 2019. - from 54-57.

6. Chemborisova N.Sh., Chernenkov I.D. Stochastic model of short-circuit currents dependence in network nodes on their balance conductivities, Collection of works of the IXth Intern. scientific and technical Conf. Energy, computer science, innovation - 2019. In 2 vols. T 1. - 2019. -P. 51-54.

7. Chernenkov I.D., Chemborisova N.Sh. Using of the balance-conductivity method during electric power system modes optimization. "Information technologies, energy and economics (electric power, electrical and heat engineering, mathematical modeling and information technologies in production). Collection of works of the XVI-th International scientific-technical Conf. stud. and grad. stud. In 3 t. T1. - 2019. Pp. 87-89.

8. Kovalev G.F., Lebedeva L.M., Voropai N.I. Electric power systems reliability. Novosibirsk: Nauka, 2015. - 224 p.

9. Mode dispatcher simulator «Finist». http://www.monitel.ru/download/Finist.pdf

10. Textbook on the course "Algorithms for power supply systems automated calculations" for students studying in the direction of "Electric power" / S.V. Shulzhenko; Ministry of education and science of the Russian Federation, National research University "MPEI". - Moscow: MPEI publishing house, 2015. - $123 \mathrm{p}$.

11. Venikov V.A., Zhuravlev V.G., Filippova T.A., "Power plants and power systems modes optimization", Moscow, Energoizdat, 1981.

12. Power Flow Test Cases [Electronic resource] Power Systems Test Case Archive. University of Washington. Access mode: http://labs.ece.uw.edu/pstca/ free.

13. Stable Renewable Plant Voltage and Reactive power control NERC ERSTF June 11-12, 2014.

14. Characteristics of Wind Turbine Generators for Wind Power Plants IEEE PES Wind Plant Collector System Design Working Group Contributing Members: E.H. Camm, M. R. Behnke, O.Bolado, M. Bollen, M. Bradt, C. Brooks, W. Dilling, M. Edds, W. J. Hejdak, D. Houseman, S.Klein, F. Li, J. Li, P. Maibach, T. Nicolai, J. Patiño, S. V. Pasupulati,N. Samaan, S. Saylors, T.Siebert, T. Smith, M. Starke, R. Walling.

15. Wind energy in Europe in 2019 Trends and statistics. Windeurope.org. Published in February 2020.

16. Fedorov V.K. The distributed generation influence on electric energy power losses and quality / V.K. Fedorov, E.N. Leonov, D.V. Fedorov // Omsk scientific bulletin. - 2016 - №6 (150) - Pp. 72-76. 
17. Maximizing the Integration of Wind Power in Distribution Systems Wind Power in Distribution Systems. Department of Energy and Environment Division of Electric power engineering. Chalmers University of technology. Goteborg, Sweden, 2013 - p. 51. 